\title{
Harmonic Analysis of Electric Vehicle Charging on the Distribution System Network with Distributed Solar Generation
}

\author{
T. Busatto, S. K. Rönnberg and M. H. J. Bollen \\ Electrical Power Engineering \\ Luleå University of Technology (LTU) \\ 931 87, Skellefteå, Sweden \\ E-mail: tatiano.busatto@Itu.se
}

\begin{abstract}
Electric vehicle chargers and solar photovoltaic inverters are two types of household loads that can potentially impact the power quality of the grid. This paper presents a view of the consequences that the connection of these two nonlinear loads into a low-voltage installation can create on voltage harmonic distortion. The analysis considers the combined impact on network impedance and current harmonic distortion. First, the network impedance for phase-to-neutral connections is obtained considering the uncertainty in customer impedance. For this, a Monte Carlo simulation and the concept of transfer impedance are used. Second, based on real measurements, the current harmonic distortion of these two nonlinear loads are used to calculate the resulting voltage distortion at any bus of interest in the network. The analysis is applied to an existing low-voltage network in Sweden. Based on the study case, results show that some harmonics may increase by about $83 \%$ as a function of the penetration of electric vehicles and photovoltaic installations.
\end{abstract}

Key words. Power system harmonics, electric vehicles, photovoltaic systems, EMI filters, Monte Carlo methods.

\section{Introduction}

Driven mainly by advances in technology and global targets to reduce greenhouse gas emissions [1], in the near future, the global fleet of conventionally-fueled vehicles will be replaced by electric vehicles (EV's).

On/off-board EV chargers or dedicated EV charging stations spread out in residential and public locations will gradually introduce a considerable number of nonlinear loads into the power system.

Parallelly, the presence of a photovoltaic installation $(\mathrm{PV})$ in residential areas can extend the environmental benefits of $\mathrm{EV}$ owners by significantly reducing the $\mathrm{CO}_{2}$ contribution associated with conventional electrical power generation and reduce the return on investment providing power to both the home and the EV.

Combining the nonlinearities from EV's and PV's, the power system will be subject to a new level of current harmonics injections. Additionally, there is an issue regarding the changes in the network impedance created by the connection of EV's and PV's into the system. These impedance changes, as shown in [2], [3], shift the harmonic resonances may causing amplification of certain harmonics and may leading to the limits to be exceeded.
The combination of both impact - harmonic currents and network impedance - faces the utility companies to new challenges in determine and mitigate voltage harmonic distortion over the network.

This paper aims to understand and quantify the impacts of EV's charging and PV's on the voltage harmonic distortion of low voltage (LV) installations. The paper is an extension of previous studies considering PV's [4], [5], including now the EV's issue and drawing attention to the fact that the penetration of both technologies are prone to create changes on the harmonic resonances.

The used methodology considers the impact of the current harmonic distortions and changes in the network impedance from customers with PV and EV to estimate resulting voltage distortion at different locations of an LVnetwork. By using a Monte Carlo (MC) simulation, the transfer impedance of an existing LV-network is first determined and combined with the injected current harmonics obtained from typical residential loads, EV's and PV's.

This study considers only single-phase PV inverters and slow EV chargers (i.e., Mode 1 and Mode 2 [6]) due to the actual predominance in residential areas [7].

The rest of the paper is organized as follow. Section 2 presents the methodology. Sections 3 analyze the EV charger input impedance. Section 4 describe the current harmonics from EVs, PVs and residential loads. Section 5 presents the analysis results and finally, Section 6 presents the conclusion.

\section{Low-voltage network}

The methodology is developed and applied to an existing low-voltage network in Sweden. The network considers a single LV feeder from a typical suburban grid with 28 customers connected to a 500-kVA transformer $(10 / 0.4 \mathrm{kV}$, Dyn $11,4.9 \%)$. The cable and transformer data, as well the network topology are described in detail in [5].

\section{Methodology}

Based on the network data, the main steps of the methodology are outlined below. 
1) Determination of the Y-bus admittance considering the network impedance and random household loads, EV and PV connections from customers using a Monte-Carlo simulation;

2) Determination of the phase-to-neutral transfer impedance matrix;

3) Determination of the resulting voltage distortion by the multiple harmonic current injections from customers;

4) Analysis of the results and comparison with EN 50160 [8] standard limits.

\section{A. Transfer impedance matrix}

As the first steps in the method, (i.e., steps 1 and 2) the Y-bus admittance matrix and the probability distribution of the phase-to-neutral transfer impedance matrix for each frequency are obtained. The method is essentially the same as that used in [5] with some modifications in order to include single-phase EV chargers into the scope. A key aspect of that study is the definition on how PV inverters and common household loads are randomly added to the grid impedance. The core uses a Monte Carlo (MC) simulation, in which input data is replicated in Table 1.

Table 1. Monte Carlo Simulation Input Data Configuration [5].

\begin{tabular}{lcc}
\hline \multicolumn{1}{c}{ Characteristic } & Distribution Function & Parameters \\
\hline Load resistance & Continuous Uniform & $\operatorname{Min}=14.4 \Omega, \max =10.6 \mathrm{k} \Omega$ \\
Load inductance & Continuous Uniform & $\operatorname{Min}=14 \mathrm{mH}, \max =500 \mathrm{mH}$ \\
Load capacitance & Normal & $\mu=5 \mu \mathrm{F}, \sigma=\mu / 3 \mu \mathrm{F}$ \\
PV capacitance & Discrete Uniform & {$[0.52 .55 .07 .510] \mu \mathrm{F}$} \\
\hline
\end{tabular}

Table 1 does not include the EV chargers impedance distribution, which will be addressed and determined in Section 4.

\section{B. Determination of the voltage harmonic distortion}

The estimation of the total voltage harmonic distortion (i.e., step 3) concerns to the aggregation rule for the absolute value of harmonics from different sources, according to IEC 61000-3-6 [9], read as follows:

$$
\left|U_{t o t}\right|=\sqrt[\alpha]{\left|U_{1}\right|^{\alpha}+\left|U_{2}\right|^{\alpha}+\cdots\left|U_{n}\right|^{\alpha}}
$$

where $\boldsymbol{\alpha}$ is the summation exponent that has a value between 1 and 2 according to Table 2 .

Table 2. Summation exponent defined in IEC 61000-3-6 for different harmonic orders.

\begin{tabular}{cc}
\hline Harmonic order & $\boldsymbol{\alpha}$ \\
\hline $\mathrm{h}<5$ & 1 \\
$5 \leq \mathrm{h} \leq 10$ & 1.4 \\
$\mathrm{~h}>10$ & 2 \\
\hline
\end{tabular}

The transfer impedance matrix relates the voltage at a certain location in the grid to the currents at a number of other locations in the grid (as well as the local current).

$$
\left|U_{j}\right|^{a}=\sum_{k}\left|Z_{j k} I_{k}\right|^{a}
$$

where the terms $\boldsymbol{Z}_{\boldsymbol{j} \boldsymbol{k}} \boldsymbol{I}_{\boldsymbol{k}}$ are the complex voltages in the same way as in the aggregation law.

Having the transfer impedance matrix, expression (2) can be used to calculate the voltage distortion due to multiple injections of harmonic currents from the customer.

\section{EV Charger Input Impedance}

From the harmonic analysis point of view, the input impedance (or equivalent impedance characteristics) seen from the EV chargers' terminals, plays an essential role in defining the impact on harmonic resonances on the power system. However, defining the exact EV chargers input impedance without knowing the circuit detail can be a difficult task due to the circuit complexity and dynamic operation. It is often only possible obtaining it by specific impedance measurement procedure. The complexity of this circuit can be reduced to an equivalent circuit model considering only the key elements that have major impact on the harmonic range.

A typical unidirectional EV charger designed to operate in slow charging mode uses a diode bridge in conjunction with an EMI filter, power factor correction and DC/DC converters circuits [10], [11]. The circuit blocks located in the AC side, have the greatest impacts on the harmonic range, as was verified through measurements in [12].

\section{A. EV charger EMI filter}

As a front end to the power system is the EMI filter, which is typically a combination of single- or multi-stage passive $\pi$-filter with one CM choke. This filter ensures that the EV charger will meet the conducted emission limits prescribed by IEC 61851-21 [6] or SAE J-J1113 [13] standards, but as a drawback, it plays a crucial role in defining harmonic resonances in combination with the rest of the grid impedance [14].

For the sake of analysis, the circuit illustrated in Fig. 1 show a typical second-order EMI $\pi$-filter which attenuate by $40 \mathrm{~dB}$ both common-mode (CM) and differential-mode (DM) noises.

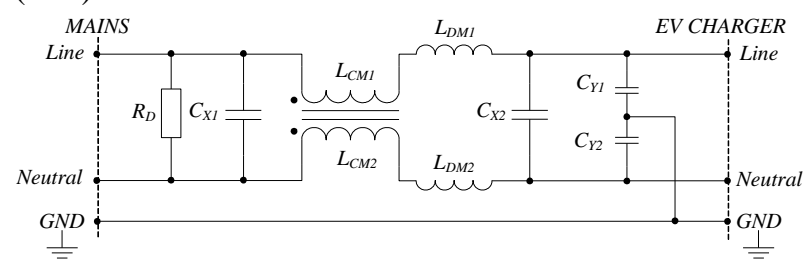

Fig. 1. Simplified EV charger single-stage EMI filter.

Since the interest is only in the driving impedance given by the single-phase connection, the circuit can be simplified to an equivalent circuit for differential mode signals as illustrated in Fig 2.

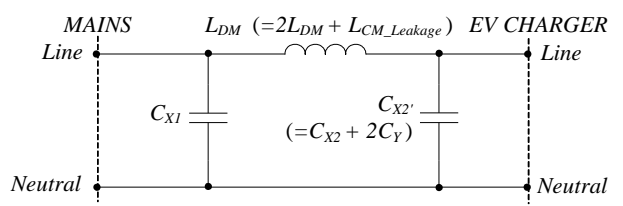

Fig. 2. EMI Filter DM equivalent circuit.

To determine the resonances, the importance relies on determining the equivalent $C_{X 1}, C_{X 2}$, capacitances and $L_{D M}$ inductance, and eventually establish a link with different EV chargers.

These values are particularly dependent on the EV charger design characteristics (e.g., switching frequency, rated power, circuit complexity, etc.) and, for instance, the values do not always have a direct relation to the $\mathrm{EV}$ charger rated power. The determination of these values is considered difficult, and there is no widely accepted design 
practice - different authors give different procedures (e.g., [15]-[17]). Even using theoretical models for the design, post-adjustments are often needed to cope with the higher frequency limits.

Although the components range can vary for different designs, capacitors and inductors should have practical values. For instance, a simple 5.0 $\mu \mathrm{F}$ line-voltage filter capacitor, or even a $20 \mathrm{mH} / 10 \mathrm{~A}$ inductor is too bulky and impractical for $\mathrm{EV}$ applications. Ultimately, the right choice of $C_{X}$ capacitor and $L_{D M}$ inductance values is about a design trade-off and fine-tuning of the filter performance.

Considering as reference the charging modes 1 and 2 and rated currents of $10 \mathrm{~A}, 16 \mathrm{~A}$, and $32 \mathrm{~A}$, a survey from filter manufacturers [18]-[20] reveals that practical $C_{X}, C_{Y}$ and $L_{D M}$ ranges as the listed values in Table 3.

Table 3. Typical inductance and capacitance values for EMI filters in the range of $10 \mathrm{~A}$ and $32 \mathrm{~A}$.

\begin{tabular}{crr}
\hline Filter element & Minimum & Maximum \\
\hline$L_{D M}$ & $0.7 \mathrm{mH}$ & $8.0 \mathrm{mH}$ \\
$C_{X}$ & $0.1 \mu \mathrm{F}$ & $1.0 \mu \mathrm{F}$ \\
$C_{Y}$ & $1.0 \mathrm{nF}$ & $4.7 \mathrm{nF}$ \\
\hline
\end{tabular}

The choice within the ranges will depend on the required filter volume and weight, and attenuation level, as well the number of filter stages.

\section{B. Impact of PV on Voltage Distortion}

To analyse the impact of $C_{X}, C_{Y}$ and $L_{D M}$ in creating resonances, consider the simplified system illustrated in Fig. 3, where a number EV chargers represented by singlestage EMI filters are connected to a point of common coupling (PCC) into the grid.

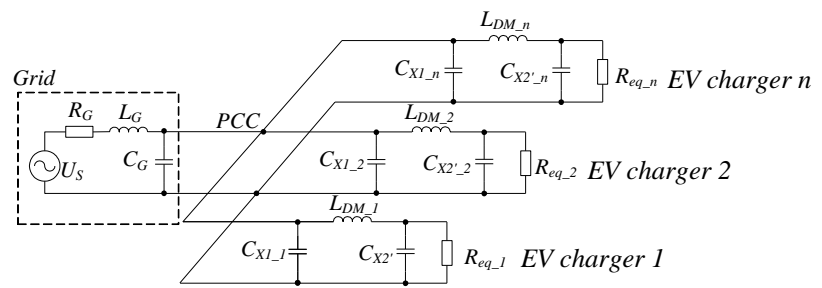

Fig. 3. Simplified schema of several EV chargers connected to a common coupling point in the grid.

In the circuit, the equivalent grid resistance, $\boldsymbol{R}_{\boldsymbol{G}}$, and inductance, $\boldsymbol{L}_{\boldsymbol{G}}$, is mainly attributed to the LV cable and the short circuit impedance of the transformer. $\boldsymbol{C}_{\boldsymbol{G}}$ is the simplified representation of the equivalent capacitance seen by the loads at PCC, while the equivalent resistance of the EV charger, $\boldsymbol{R}_{\boldsymbol{e q}}$, is dependent on the rated power and battery charging state [21].

A simple analysis from the circuit reveals the possible emergence of various resonances at PCC. For instance, in a system with $\boldsymbol{N}$ different EV chargers, each one with a $\boldsymbol{n}$ stages EMI filter, the resulting number of resonances, $\mathbf{M}$, can be approximated by:

$$
\mathrm{M}=1+\sum_{i=1}^{N} 2 n_{i}
$$

The first and most important is the series resonance created by the combination of the grid equivalent inductance, $L_{G}$, and capacitance, $C_{G}$, and the sum of the capacitors $C_{X I}$ from the individual chargers. The simplified expression yields to:

$$
f_{r e s 1}=\frac{1}{2 \pi \sqrt{L_{G}\left(C_{G}+\sum_{n=1}^{N} C_{X 1 n}\right)}}
$$

The other resonances have less dependence on the grid inductance and can vary from charger to charger. One is a series resonance created by the combination of $L_{D M}$ and $C_{X 2}$, branch, and the other is the parallel resonance created by the loop $L_{D M},\left(C_{G}+C_{X I}\right)$, and $C_{X 2}$. Both resonances will range from $1.8 \mathrm{kHz}$ up to $19 \mathrm{kHz}$ using the listed values in Table 3, being the last, slightly impacted by the equivalented grid capacitance, $C_{G}$. The simplified expressions of these resonance frequencies are given by:

$$
\begin{gathered}
f_{\text {res } 2}=\frac{1}{2 \pi \sqrt{L_{D M} C_{X 2 \prime}}} \\
f_{r e s 3}=\frac{1}{2 \pi \sqrt{L_{D M} \frac{\left(C_{G}+C_{X 1}\right) C_{X 2 \prime}}{C_{G}+C_{X 1}+C_{X 2 \prime}}}}
\end{gathered}
$$

Compared to $\boldsymbol{f}_{\text {res } 1}$, the resonances $\boldsymbol{f}_{\text {res } 2}$ and $\boldsymbol{f}_{\text {res } 3}$ present a sharp attenuation given by the equivalent impedance from the rest of the charger. Resonances $\boldsymbol{f}_{\text {res2 } 2}$, $\boldsymbol{f}_{\text {res } 3}$, are attenuate about 800 to 5000 times more than $\boldsymbol{f}_{\text {res } 1}$, depending mainly on the relation between transformer and cables resistance, and EV charger equivalent resistance.

Considering a hypothetical system with three equal EV chargers with rated power $3.3 \mathrm{~kW}, \boldsymbol{L}_{\boldsymbol{D} \boldsymbol{M}}$ and $\boldsymbol{C}_{\boldsymbol{X}}$ equal to $1.0 \mathrm{mH}$ and $1.0 \mu \mathrm{F}$, respectively, connected to a distribution network supplied by a $500 \mathrm{kVA}$ transformer with $\boldsymbol{C}_{\boldsymbol{G}}$ equal to $140 \mu \mathrm{F}$, the resulting resonances

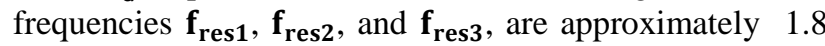
$\mathrm{kHz}, 5.0 \mathrm{kHz}$ and $5.1 \mathrm{kHz}$. However, only $\mathbf{f}_{\text {res } 1}$ has significant magnitude, while $\mathbf{f}_{\text {res2 }}$, and $\mathbf{f}_{\text {res3 }}$ are very close to each other and they can only be noticed in an eventual drop of the equivalent resistance of EV charger, which in theory can happen, especially when the battery is close to full charge.

Summarizing, only $\mathbf{f}_{\text {res1 }}$ has potential impact on harmonic resonances and it is directly dependent on $C_{X I}$, consequently, in this study, a continuous distribution probability function from $0.1 \mu \mathrm{F}$ up to $1.0 \mu \mathrm{F}$, will be considered as input for the Monte Carlo simulation.

\section{Harmonics from Domestic Customers}

\section{Harmonics from common household equipment}

Current harmonics are injected into the grid, not only by PV inverters and EV chargers but from common household equipment as well (e.g., computers, microwave ovens, energy-efficient lights to name a few). To set a reference for harmonic injections, the current harmonics were measured at the delivery point for a number of customers, without PV installation and EV. Fig. 4 shows the 95th percentile (CP95) of the current harmonics for the three individual phases for three different customers measured over one week, 10 minutes average. 

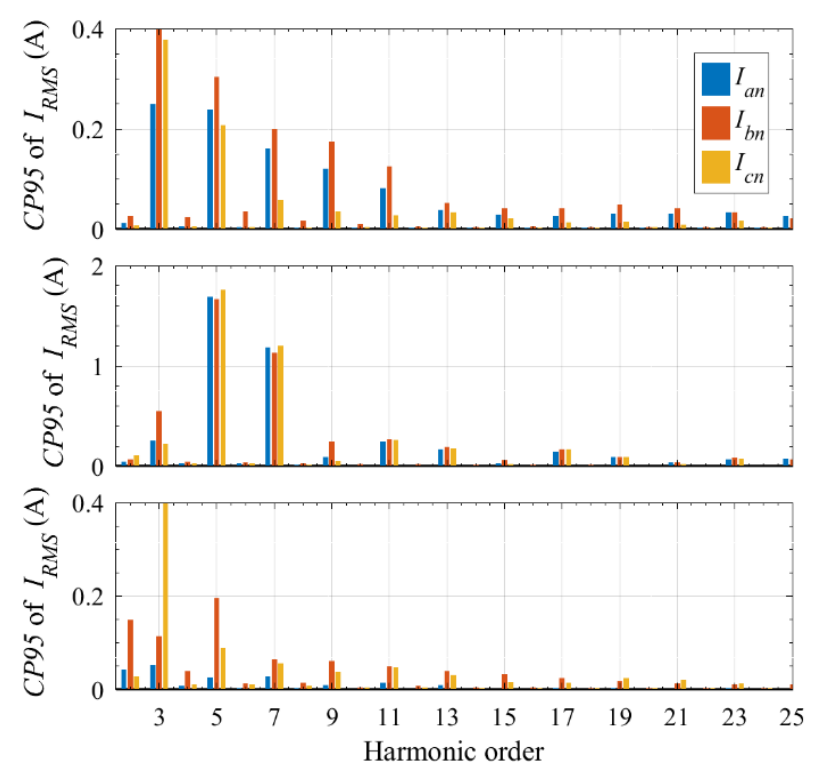

Fig. 4. CP95 of the current harmonics measured at customer A. (top), B (middle), and C (bottom) over one week.

As can be seen in Fig. 4 the current harmonic distortion varies between customers and between phases. The spectra and corresponding magnitudes depend on what type of equipment a customer has connected at that certain moment in time.

\section{Harmonics from $E V$ 's and $P V$ 's}

The representative set of EV's and PV's current harmonic distortions are shown in Fig. 5. EV's and PV's current harmonic distortions.
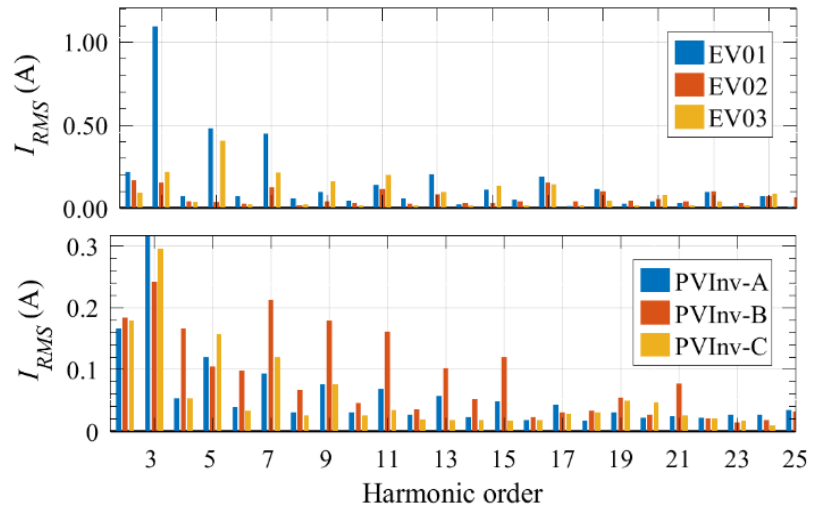

Fig. 5. EV's and PV's current harmonic distortions.

The current harmonics of three EV's refers to a snapshot of 10 seconds window performed on the phase-neutral connection of the charger in the Pehr Högström laboratory with Luleå University of Technology in Skellefteå. EV01 and EV02 are Full Electric Vehicles, while EV3 is a Hybrid Plug-in Vehicle.

The current harmonics from PV inverters were measured at TU Dresden laboratories, further details of the inverters are listed in [22]. The set covers some of the inverter topologies used in small residential and commercial applications.

Note the difference in the current scale of EV and PV Inverters current harmonics in comparison with the common household equipment shown in Fig. 4. With exception of EV01 and customer C, harmonics pattern does not differ significantly among the measurements.

\section{Results}

Table 4 lists the five scenarios used to evaluate the results in terms of $\mathrm{EV}$ and $\mathrm{PV}$ penetration. A scenario without PV and EV chargers is considered as reference for the analysis.

Table 4. EV and PV penetration scenarios.

\begin{tabular}{crr}
\hline Scenario & PV penetration & EV penetration \\
\hline REF & $0.0 \%$ & $0.0 \%$ \\
PV100-EV000 & $100.0 \%$ & $0.0 \%$ \\
PV000-EV100 & $0.0 \%$ & $100.0 \%$ \\
PV050-EV050 & $50.0 \%$ & $50.0 \%$ \\
EV100-PV100 & $100.0 \%$ & $100.0 \%$ \\
\hline
\end{tabular}

The two customer ends 01-an and 14-bn, empirically selected, are used to verify possible voltage distortion and source impedance variations among customers. Throughout this paper we use the term customerconnection to refer to these ends, where the number refers to the customer bus ID in the LV feeder and the letters to the phase terminals (e.g., an means an impedance or voltage harmonic result considering a connection between the phase- $a$ and neutral terminals as reference).

\section{A. Impact on source impedance}

Considering 250 samples for MC simulation, Fig. 6 and Fig. 7 show the CP95 of source impedance magnitude for the two customers for the different scenarios.

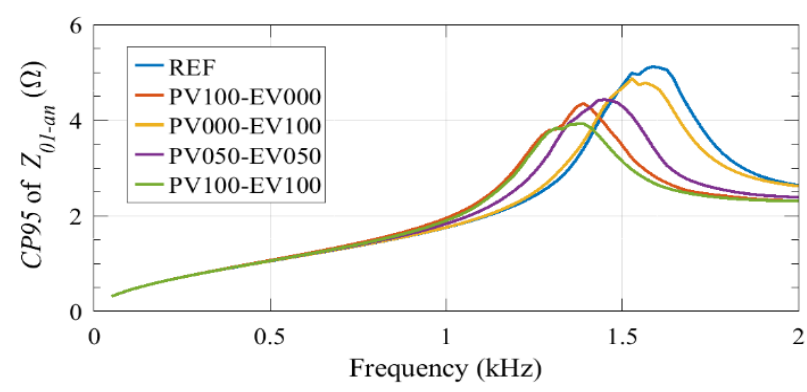

Fig. 6. Source impedance magnitude 01-an.

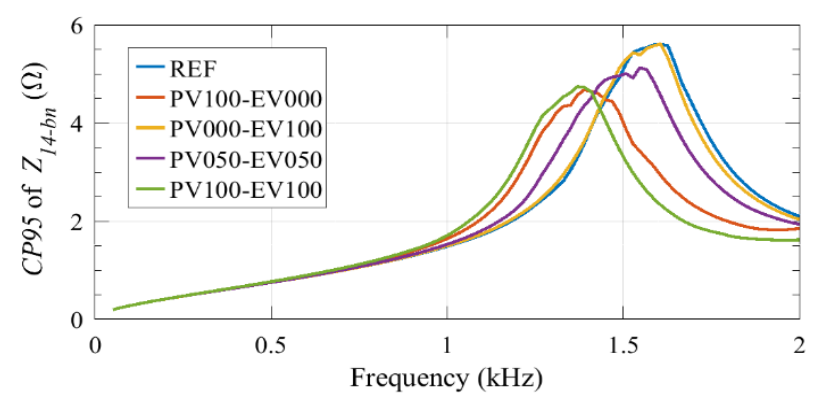

Fig. 7. Source impedance magnitude 14-bn.

From Fig. 6 and Fig. 7, it can be observed that when there is no PV inverters and EV chargers connected to the grid, the dominant resonance is located at about $1.6 \mathrm{kHz}$ with a magnitude between $5.1 \Omega$ and $5.6 \Omega$. The major change in the resonance happens when $100 \% \mathrm{PV}$ and EV charger penetration is considered. In this case, the resonance shifts about $250 \mathrm{~Hz}$ to a lower frequency band and the magnitude decrease about $20 \%$ depending on the customer location. Due to the lower capacitance, the impact of the EV chargers on the resonance is proportionally smaller compared to PV. However, the results in Fig. 6 and Fig. 7 indicate a variation in the resonance frequency and magnitude as a function of 
different EV's penetration levels. This is most evident for the results of the customer-connection 01-an (Fig. 6), where the difference in the resonance magnitude between the scenario REF and PV000-EV100 or between the scenario PV100-EV000 and PV100-EV100 is about $5 \%$ and difference in the frequency of about $50 \mathrm{~Hz}$.

\section{B. Impact on individual voltage harmonic distortion}

In Fig. 8 and Fig. 9 the individual voltage harmonics calculated using the expressions described in Section 3.B are presented. Both figures refer to the customerconnection 01-an. Fig. 8 and Fig. 9 show the results for REF and EV100-PV100 scenario, respectively.

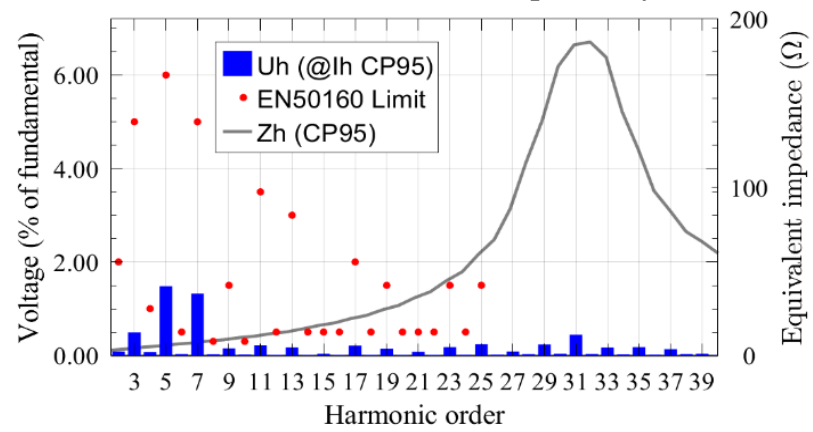

Fig. 8. Harmonics voltage for customer-connection 01-ab for scenario REF.

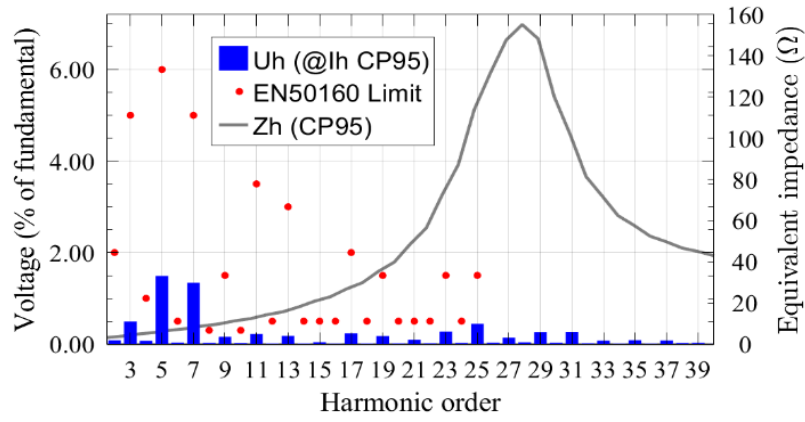

Fig. 9. Harmonics voltage for customer-connection $01-\mathrm{ab}$ for scenario PV100EV100.

In the figures, the equivalent impedance is the sum of all transfer impedance between the other customer ends and the customer end under analysis. The red dots are the individual voltage harmonic limits given by EN50160 [8]. The current harmonics injections for the single-phase PV inverters and $\mathrm{EV}$ chargers are in the same phases where the random capacitances are connected, being the choice of the individual devices randomly selected from the harmonics dataset described in Section 5.D.

Under normal conditions the impact of the main resonance is most significant for the upper frequency range (e.g., the harmonic 31 is amplified by the harmonic resonance). The shift to a lower resonant point compared to Fig. 8 is seen in Fig. 9. The resonant frequency in this case impacts more the harmonic within the range 23-29. For the odd harmonics, the biggest amplification is at h25 which increases with $83 \%$. Worth emphasizing is that even with this amplification the magnitude only reaches $0.44 \%$ of the fundamental which is well below the limit at $1.5 \%$ define by the standard. However, since EN50160 defines the voltage harmonic limits for the global contribution of the LV installation, the harmonic resonance shifting to the lower frequency range decreases the remaining margin below the limit. Assuming installations with higher levels of background distortion, the limits could be exceeded.

In Fig 11 the impact on the 29th harmonic as PV inverters are added into the network is shown as an example. In the graph, phases are represented by a set of three dots, and different dot colors are used to distinguish the different customers.

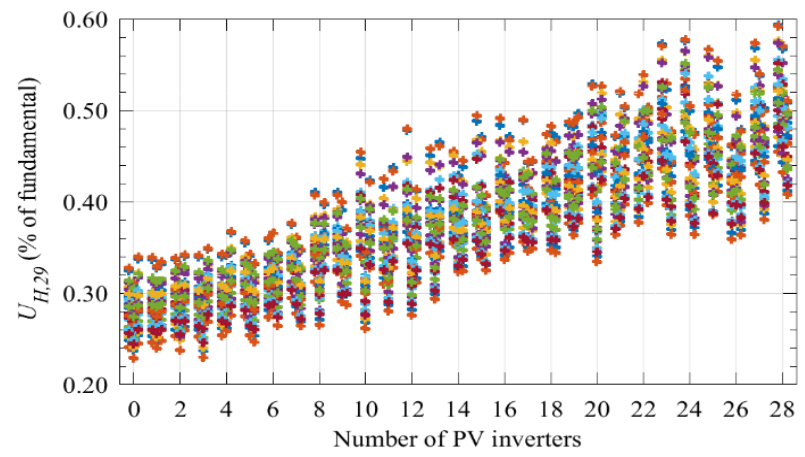

Fig. 10. Voltage harmonic H29 considering the increasing of $\mathrm{PV}$ inverter penetration for customer-connection 01-an.

Results show that an increasing number of PV's in the system can promote an increase of about $78 \%$ on the 29 th harmonic on some customer-connections. The maximum value of $0.59 \%$ of fundamental is reached when $28 \mathrm{PV}$ 's are considered, but not always the higher number of PVs means a higher value. For instance, the maximum value with 24 PV's is higher than when 26 PV's are considered. Also, different customer-connections have different sensitivity to the number of PV's.

\section{A. Impact on voltage Total Harmonic Distortion (THD)}

Although variations in individual harmonics occur, result from Fig. 11 shows that there are no significant changes in the THD\% as the PV's are added to system and values for all customers are well below the limit.

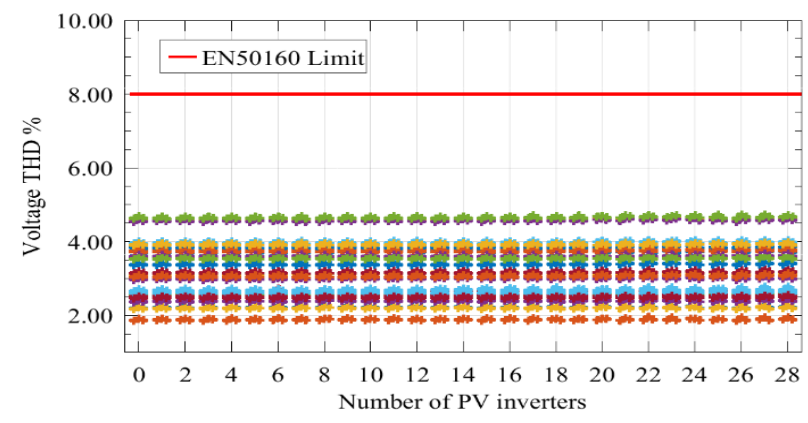

Fig. 11. Voltage THD\% considering the increasing of PV inverter penetration for customer-connection 01-an

\section{General overview of the impacts}

Fig. 12 shows an overview of voltage harmonic distortion for customer-connection 01-an under different scenarios.

The impact of the connection of PV's and EV's is most significant for the higher frequency range as can be seen by the differences in magnitude compared to the REF scenario. The reason is that the penetration of PV's and EV's increase the impedance magnitude as shown in Fig. 9. On the other hand, assuming that the summation exponent at higher harmonic orders is lower, more cancelation effect is achieved at the higher harmonic orders, reducing the overall impact. Harmonic orders below 15 are almost not impacted by the connection of PV's and EV's. In general, for the considered network and 


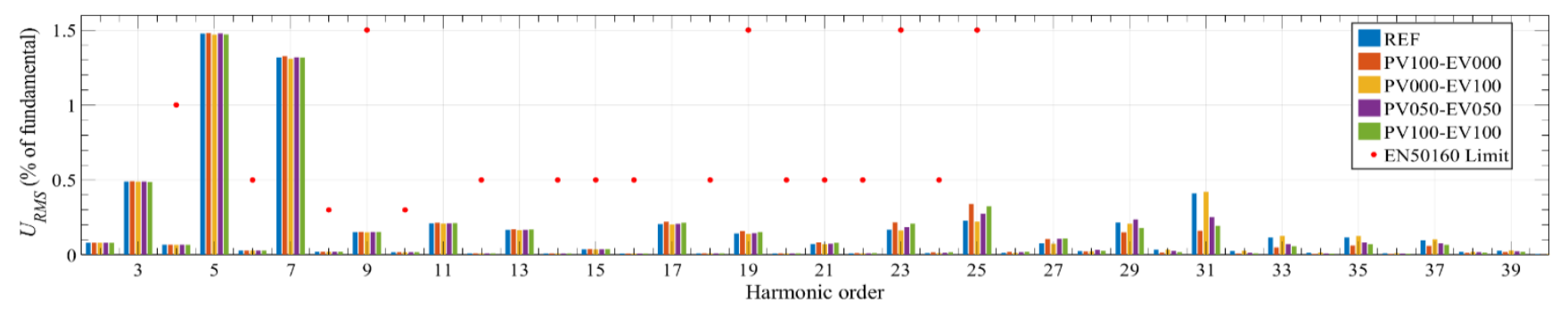

Fig. 12. Voltage harmonic distortion for customer-connection 01-an under different scenarios.

load characteristics, there is no risk of the voltage harmonics to be above the EN50160 [8] limits, but as the number of customer increase, the resonance can potentially shift to the lower order harmonics.

\section{Conclusion}

This work has discussed an analysis method to address the harmonic resonances present in public low-voltage networks considering the penetration of PV and EV. The results have shown that the main impact of a large-scale introduction of PV's and EV's is on harmonic resonance.

Although it was shown that some harmonics may increase as a function of the penetration of EV's and PV's, the values are still well below the standard limits in this specific network. However, in LV grids with less headroom for an increased level of harmonics, this needs to be considered before a large-scale introduction of PV and EV. Depending mainly on the number of customers, there is the risk that the resonant is shifted to the frequency range where the highest magnitudes of current harmonics are found (h3 to h9). In the lower frequency range, there is also less cancelation effect due to differences among the harmonic phase angles, which can add to the amplification. The total harmonic distortion value is not significantly impacted by the change in impedance which indicates that while some harmonics are amplified, others are reduced.

The study does not fully cover the time-dependence and connection concurrency of household loads, EV's, and PV's, which can lead to an overestimation of the results. The background voltage distortion, commonly present in LV-networks is not considered in this study, which can lead to an underestimation of the actual results. A further study with more focus on these issues is therefore suggested.

\section{References}

[1] United Nations, "Emissions Gap Report," Katowice, 2018.

[2] J. Meyer, P. Schegner, R. Stiegler, I. Röder, and A. Belger, "Harmonic Resonances in Residential Low Voltage Networks Caused by Consumers Electronics," in 24th International Conference on Electricity Distribution, 2017, pp. 1-5.

[3] H. L. M. Monteiro et al., "Harmonic impedance measurement based on short time current injections," Electr. Power Syst. Res., vol. 148, pp. 108-116, Jul. 2017, doi: 10.1016/j.epsr.2017.03.031.

[4] T. Busatto, S. Rönnberg, and M. H. J. Bollen, "Photovoltaics and Harmonics in Low-Voltage Networks," no. 473. Energiforsk AB, p. 60, 2018.

[5] T. Busatto, A. Larsson, S. K. Ronnberg, and M. H. J. Bollen, "Including Uncertainties from Customer Connections in Calculating Low-Voltage Harmonic Impedance," IEEE Trans. Power Deliv., vol. 34, no. 2, pp. 606-615, 2019, doi: 10.1109/TPWRD.2018.2881222.

[6] IEC 61851-1, "Electric vehicle conductive charging system - Part 1: General requirements." IEC, 2017.
[7] "Global EV Outlook 2018 - Towards Cross-modal Electrification," 2018.

[8] EN 50160, "Voltage characteristics of electricity supplied by public electricity networks." CENELEC, p. 40, 2010.

[9] IEC 61000-3-6, "Assessment of emission limits for the connection of distorting installations to MV, HV and EHV power systems." IEC 61000-3-6 Ed. 2, 2008.

[10] M. Yilmaz and P. T. Krein, "Review of battery charger topologies, charging power levels, and infrastructure for plug-in electric and hybrid vehicles," IEEE Trans. Power Electron., vol. 28, no. 5, pp. 2151-2169, 2013, doi: 10.1109/TPEL.2012.2212917.

[11] L. Rubino, C. Capasso, and O. Veneri, "Review on plug-in electric vehicle charging architectures integrated with distributed energy sources for sustainable mobility," Appl. Energy, vol. 207, pp. 438-464, 2017, doi 10.1016/j.apenergy.2017.06.097.

[12] T. Busatto, V. Ravindran, A. Larsson, and M. H. J. Bollen, "Estimation of the consumer electronics capacitance for harmonic resonance studies by a non-invasive measurement method," Proc. Int. Conf. Harmon. Qual. Power, ICHQP, vol. 2018-May, pp. 1-6, 2018, doi: 10.1109/ICHQP.2018.8378869.

[13] SAE J-1113, "Electromagnetic Compatibility Measurement Procedures and Limits for Components of Vehicles, Boats (up to $15 \mathrm{~m}$ ), and Machines (Except Aircraft) $(16.6 \mathrm{~Hz}$ to $18 \mathrm{GHz}) . " 2013$.

[14] A. Di Napoli and A. Ndokaj, "EMC and safety in vehicle drives," Proc. 2011 14th Eur. Conf. Power Electron. Appl. EPE 2011, pp. 1-8, 2011.

[15] R. L. Ozenbaugh, EMI Filter Desin. Marcel Dekker, Inc, 1995.

[16] F. Y. Shih, D. Y. Chen, Y. P. Wu, and Y. T. Chen, "A procedure for designing EMI filters for AC line applications," IEEE Trans. Power Electron., vol. 11, no. 1, pp. 170-181, 1996, doi: 10.1109/63.484430.

[17] K. Kostov, V. Tuomainen, J. Kyyrä, and T. Suntio, "Designing power line filters for DC-DC converters," in 11th International Power Electronics and Motion Control Conference (EPE-PEMC 2004), 2004, no. September, p. 6.

[18] Würth Elektronik, "WE-CLFS Line Filter." [Online]. Available: https://katalog.we-online.com. [Accessed: 12Oct-2019].

[19] Schaffner, "Single-phase, single stage AC/DC EMI Filters." [Online]. Available: https://www.schaffner.com/products/emcemi/. [Accessed: 12-Oct-2019].

[20] Delta Electronics Inc, "EMI Filters." [Online]. Available: https://www.deltaww.com. [Accessed: 12-Oct-2019].

[21] Y. Liu, Y. Li, J. Ren, S. Wang, and L. Li, "A Data-driven Harmonic Modeling Method for Electric Vehicle Charging Station," in 25 th International Conference on Electricity Distribution CIRED, 2019, no. June, pp. 3-6.

[22] R. Langella, A. Testa, J. Meyer, F. Moller, R. Stiegler, and S. Z. Djokic, "Experimental-Based Evaluation of PV Inverter Harmonic and Interharmonic Distortion Due to Different Operating Conditions," IEEE Trans. Instrum. Meas., vol. 65, no. 10, pp. 2221-2233, 2016, doi: 10.1109/TIM.2016.2554378. 